\title{
Exploitation of Gas Sensor Dynamics by the use of Echo State Networks
}

\author{
Hans-Ulrich Kobialka ${ }^{1}$, Andreas Walte ${ }^{2}$ \\ ${ }^{1}$ Fraunhofer IAIS, Schloss Birlinghoven, 53754 Sankt Augustin, Germany \\ hans-ulrich.kobialka@iais.fraunhofer.de \\ ${ }^{2}$ AIRSENSE Analytics GmbH, Hagenower Str. 73, 19061 Schwerin, Germany \\ walte@airsense.com
}

\begin{abstract}
:
Gas sensor arrays generate sensor data time series containing temporal information like gradients, noise patterns, and delays. We want to exploit temporal dynamics in gas sensor data for improving gas measurement in difficult environments, e.g. freight containers. For supervised training of pattern matching models we use Echo State Networks, a new approach for training large recurrent neural networks.
\end{abstract}

Keywords: Gas sensor arrays, ion mobility spectrometer, neural networks, time series analysis.

\section{Introduction}

Quantification of gases could be difficult in the presence of other chemical substances also influencing the sensor signal. Interpretation of such distorted sensor signals is challenging, and the risk of either false alarms or false negatives is increased.

A sensor signal contains also important information in time, for example, a metal oxide sensor may respond to different gases with the same amplitude but the response to one gas may be faster than to the other. Or, the speed of getting through a membrane may differ for different gases, and therefore sensor signal arrival time differs too. Furthermore, the gradient of sensor response usually carries additional information.

Sensor arrays are used to detect substances by combining the capabilities of different sensors, thereby increasing both the reliability of detection, and the range of detectable substances. The portable gas detector array GDA2, which we use for the work described in this paper, consists of an ion mobility spectrometer (IMS), a photoionization detector (PID), two metal oxide sensors (MOS) and an electrochemical cell (EC).

Each single sensor may respond to a gas with some characteristic pattern in time. Of course, correlations between time events observed from different sensors should be analyzed, and time differences between such events should be incorporated into this analysis too.

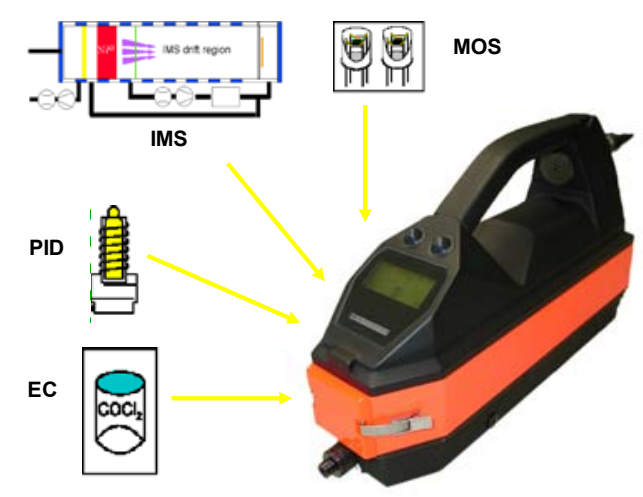

Fig. 1: The portable gas detection array GDA2 by AIRSENSE Analytics $\mathrm{GmbH}$

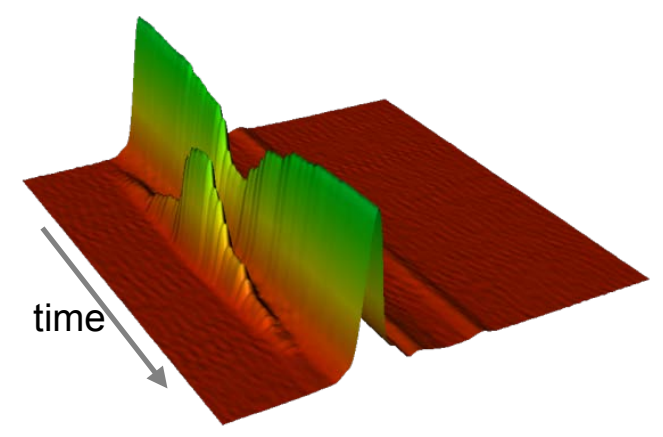

Fig. 2: An IMS spectrum of Methyl Bromide as it changes over time while varying the flow of clean air. 


\section{Quantification of Gases using an IMS}

Quantification using an IMS is a particular challenge. In short, the quantity of a gas can't be deduced just from the corresponding peak in the IMS spectrum. Instead, all gases present in the atmosphere, interact within the IMS. This has to be considered for proper quantification. A detailed explanation follows below.

The atmosphere in freight containers is usually a complex mixture of different gases, containing industrial chemicals and sometimes also fumigants.

Contaminants in higher concentrations might affect the detection of toxic compounds. Depending on the proton affinity, or respectively the electronegativity, of the contaminants in comparison to the toxic compound the product ion formation is suppressed or not. In mixtures the molecules with the highest proton affinities will be formed predominantly [2].

Due to the complex chemical reactions it is difficult to generate quantitative measurements. Depending on the humidity of the drift gas the size of the reactant ions (which consist of water clusters) changes. At the same time also its proton affinity or electronegativity changes. The intensity of the interesting product ions (i.e. the peaks in the IMS spectrum) depends on

- the concentration of the different compounds in the mixture,

- the proton affinities or electro negativities of each compound, and

- the reactions with the reactant ions.

There is a vast amount of gas mixtures which can be encountered in freight containers. Their interaction during IMS measurement can only be captured empirically, i.e., by a large amount of measurements in real environments.

For all measurements, the concentration of the interesting compounds has to be known. In our case the identification and concentration is determined by exhaustive laboratory measurements by gas chromatography mass spectrometry (GCMS).

By knowing the gases and their concentration within measurements, and the response of the IMS and the other sensors, we construct the reverse mapping, i.e., from sensor data (time series of recorded IMS spectra, and MOS, PIC and EC sensor values) to gas concentrations. This can be learned by an artificial neural network.

\section{Echo State Networks}

Echo State Networks (ESNs) is a new approach of training recurrent neural networks (RNNs). The advantage of ESNs is that only the connections to the output nodes are computed during training. This is done using regression which is far more simple and stable than compared to gradient-based methods. This simplicity enables the use of large nets having 1000 internal nodes and more. These large nets have the capacity of learning highdimensional temporal patterns.

ESNs can be used to learn system models from time series data. An ESN is continuously fed with input signals (from the time series to be analyzed). Because of feedback loops in the network, previously received input signals are still represented in the net. This kind of memory enables an ESN to do pattern matching on time series.

Echo State Networks are well recognized because of publications in well-known international journals like ,Science' and ,Neural Networks' [3] [4]. Fraunhofer holds international patents on ESNs (Europe, USA, Japan, and Canada).

\section{Experiments and Results}

The task is to quantify certain toxic gases in freight containers [1]. Freight containers may contain all possible kinds of goods and gases emitted by them. The sensor signal also contains the influence of this gas mixture present in a container, thus the signal-to-noiseratio could be bad. As a consequence, gas detection has to be robust with regard to many kinds of environmental noise.

Measurements with GDA2 were conducted at freight containers. Furthermore, air samples taken from freight containers are taken to a laboratory, some of them were mixed with Methyl Bromide (2 ppm and $7 \mathrm{ppm}$ ) and Acetone $(0.1 \mathrm{ppm})$, and then all were measured using GDA2. The collected sensor data is separated into a training set (used to create a pattern matching model using ESNs) and a test set (for evaluating how the learned model performs on unseen data).

The IMS signal is coded as a histogram of 50 bins, both for the positive and negative IMS spectra. Together with the signals from the two MOS, the PID, the EC, and the currently used dilution (additional air flow for preventing too high gas concentrations to be exposed to the sensors), this results to 105 values per time step. Depending on the duration of the measurements, time series of 17 to 19 time steps are recorded. 305 measurements are 
evaluated by leave-one-out cross-validation (LOOCV) yielding a mean absolute error of 0.63 ppm for Methyl Bromide (Figure 3).

During analysis of the data, it turned out that some container atmospheres contained high concentrations of other gases. Furthermore we found out that the sampling bags we used contained residuals which significantly influenced the IMS. This shows that quantification has been performed under difficult conditions.

\section{Conclusions}

Quantification of gases in complex atmospheres (freight containers) using an IMS is a big challenge because of complex chemical interactions within the IMS.

Within the GDA2 gas detector array, an IMS is combined with two MOS, a PID, and an EC. Therefore, in addition to the IMS analysis, sensor data fusion has to be performed.

Furthermore delays between sensors, gradients of sensor response, etc. have to be considered, thus introducing the need for time series analysis.

We tackled all three aspects (chemical interactions within the IMS, sensor data fusion, and time series analysis) by a new approach for training large recurrent neural networks. This approach, Echo State Networks, shows promising preliminary results.

In the Optima project, further measurements will be conducted to create a sound statistical basis on atmospheres of freight containers. This will be the basis for learning robust models for quantifying gases contained in freight containers.

\section{Acknowledgement}

The work presented in this paper is part of the Optima project supported by the German Federal Ministry of Research (BMBF) under the KMU Innovativ program.

\section{References}

[1] Baur X., Poschadel B., Budnik L.T.: High frequency of fumigants and other toxic gases in imported freight containers - an underestimated occupational and community health risk, Occupational \& Environmental Medicine, 67:207212, 2009.

[2] Eiceman, G.A., Karpas, Z.: Ion Mobility Spectrometry. Second Edition. Taylor \& Francis, 2005.

[3] Jaeger H. Haas H.: Harnessing Nonlinearity: Predicting Chaotic Systems and Saving Energy in Wireless Communication. Science, April 2, pp 7880, 2004.

[4] Jaeger, H., Maass, W., Principe J. (eds): Special Issue Echo State Networks and Liquid State Machines, Neural Networks, Volume 20, Issue 3, pp 287-432, 2007

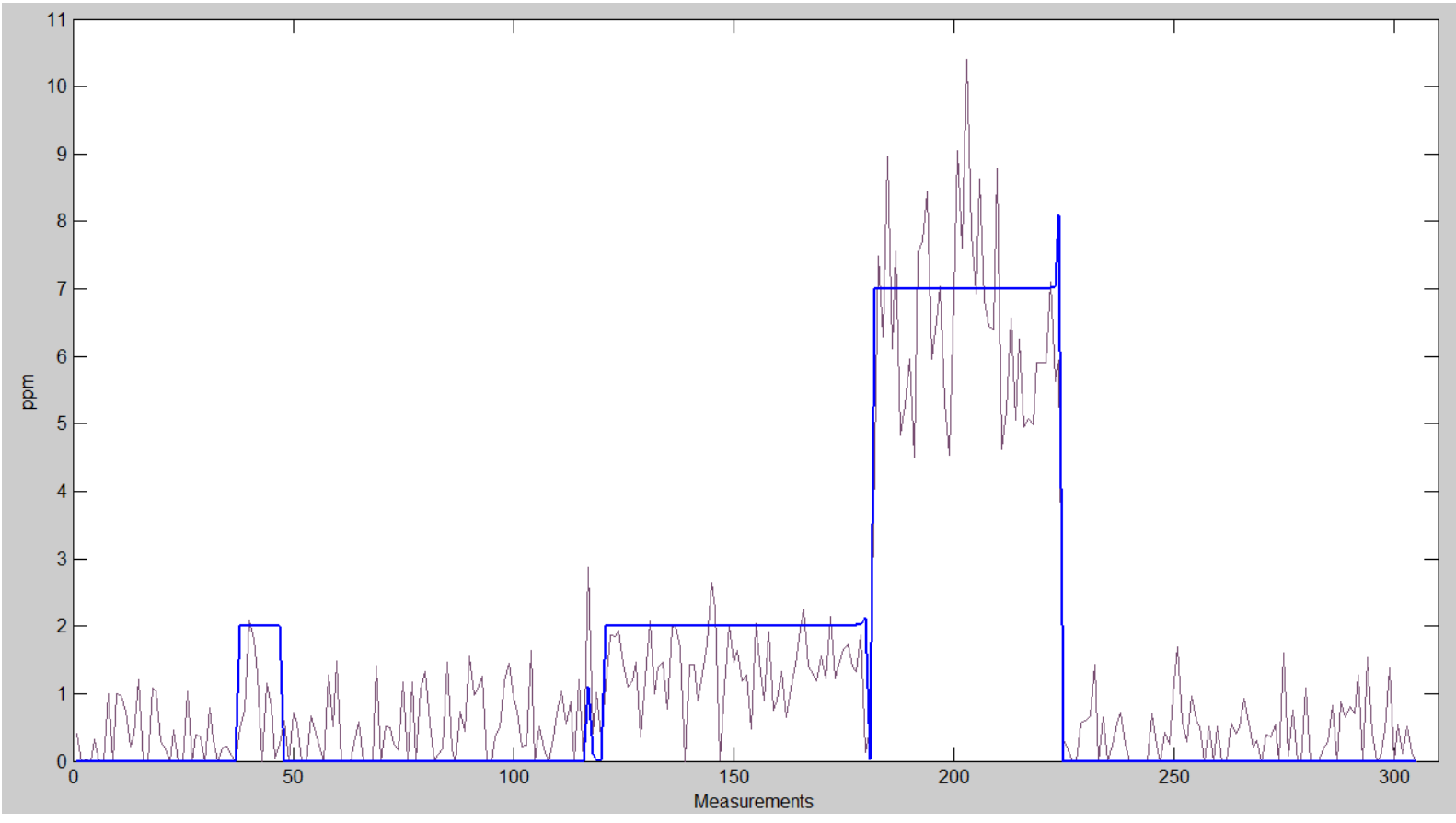

Figure 3: Methyl Bromide concentrations contained in 305 measurements (thick line) and the values estimated by an Echo State Network (thin line). 